\title{
Human African trypanosomes: challenges posed to the human immune system
}

\author{
Wellington A. OYIBO ${ }^{1 *}$, Chimere AGOMO ${ }^{1,2}$, Oladipo O. OLADOSU ${ }^{1}$, \\ Ojuromi O. TESLIM ${ }^{3}$, Adekunle O. SANYAOLU ${ }^{4}$, Jane AJULUCHUCKWU ${ }^{5}$, \\ Adetayo F. FAGBENRO-BEYIOKU ${ }^{1}$ and Ijeoma OTIGBUO ${ }^{6}$ \\ ${ }^{I}$ Tropical Diseases Research Laboratory, Department of Medical Microbiology and Parasitology, College of \\ Medicine, University of Lagos, Lagos, Nigeria. \\ ${ }^{2}$ Department of Biochemistry, Nigerian Institute of Medical Research, Yaba, Lagos, Nigeria. \\ ${ }^{3}$ Department of Zoology, Faculty of Science, Lagos State University, Ojo, Lagos, Nigeria. \\ ${ }^{4}$ Department of Global Health, College of Public Health, University of South Florida, Tampa, FL. USA. \\ ${ }^{5}$ Department of Medicine, College of Medicine, University of Lagos, Nigeria. \\ ${ }^{6}$ Department of Biology, Montgomery College, Takoma Park/Silver Spring, Maryland, USA. \\ "Corresponding author, Tel: 234-8035374004, E-mail: wellao@yahoo.com
}

\begin{abstract}
Human infection by either Trypanosoma brucei gambiense (Tbg) or Trypanosoma brucei rhodesiense $(\mathrm{Tbr})$ and the establishment of disease is made possible by the intermittent switching of their variant surface glycoprotein (VSG) and expression of serum resistance associated (SRA) protein (by Tbr) which nullifies the lytic action of the normal human serum. The ability to switch expression from one VSG to the other is recognized to be the major mechanism that permits the parasite to evade the otherwise efficient host antibody, hence preventing parasite elimination and allowing the establishment of a chronic infection. These changes were reported to: disable the host's capacity to mount a protective anti-parasite antibody response and prevent the development of effective B-cell memory against encountered variant antigenic parasite types (VATs). Both $\mathrm{B}$ cell-mediated antibody response and the Th1 cell responses leading to the production of interferon-gamma (IFN- $\gamma$ ) are required for maximum host resistance to trypanosomes, with IFN- $\gamma$ acting to induce macrophage trypanolytic and trypanostatic activities. High levels of both tumor necrosis factor alpha (TNF $\alpha$ ) and interleukin 10 (IL-10) have been associated with trypanosomal infection. Trypanosomal genetics (including the parasite's intrinsic characteristics), human immune response polymorphisms and geographical locations are important elements that describe the severity or mildness of HAT. As the parasite devices ways to evade the human immune system, and in the absence of a suitable vaccine, surveillance, prompt diagnosis and treatment with available drugs and vector control efforts will go along way in reducing the incidence of HAT.

(C) 2009 International Formulae Group. All rights reserved.
\end{abstract}

Keywords: African trypanosomes, Variant surface glycoprotein, Serum resistance associated (SRA) protein, Trypanosomal genetic polymorphisms, Immune response polymorphisms.

\section{INTRODUCTION}

The human African trypanosomiasis (HAT) commonly referred to as African sleeping sickness has been acclaimed over several decades to be more deadly than other vector-borne diseases such as malaria because death is inevitable if an infected patient is not treated promptly. Sleeping sickness is caused by two sub species of African trypanosomes:
Trypanosoma brucei rhodesiense (Tbr) [the East African sleeping sickness], and Trypanosoma brucei gambiense (Tbg) that causes the West African sleeping sickness. The parasites invade the central nervous system and the infected individual suffers from white matter encephalitis with concomitant psychiatric and motor disorders (Nikolskaia et al., 2006; Simarro et al., 2008). 
HAT caused by $T$. $b$. rhodesiense is more acute and progresses more rapidly to the late stage than $T$. b. gambiense infection.

Trypanosomes undergo antigenic variation by changing their surface coat when a new VSG gene, from a library of up to $10^{3}$ different surface antigen genes, is expressed effectively ensuring that the host immune response does not fully eliminate the organisms. This cyclical pattern of trypanosome outgrowth and variant specific elimination may continue throughout infection until the host succumbs. Thus, by switching VSG genes and expressing a new surface coat, trypanosomes evade B- and Th-cell immune responses directed against VSG epitopes displayed in the former surface coat. The end result is that new variant antigenic types (VATs) populate the vascular and extravascular tissues and perpetuates the infection, whereas trypanosomes expressing the former coat are destroyed. There is therefore a dynamic anti-VSG response by the $\mathrm{B}$ and $\mathrm{T}$ helper cells, which is directed against every new epitope formed by the parasite (Cardoso de Almeida et al., 1983).

The two subspecies ( $T b g$ and $T b r$ ) are morphologically indistinguishable microscopically and can be distinguished genetically by the human serum-associated (SRA) gene which defines T.b. rhodesiense and is found to play a significant functional role in human infectivity (Vanhamme et al., 2003). Both B cell-mediated antibody response and the Th1 cell responses leading to the production of interferon-gamma (IFN- $\gamma$ ) are required for maximum host resistance to trypanosomes, with IFN- $\gamma$ acting to induce macrophage trypanolytic and trypanostatic activities. High levels of both tumor necrosis factor alpha $(\mathrm{TNF} \alpha)$ and interleukin 10 (IL-10) have been associated with trypanosomal infection (MacLean et al., 2001, 2004, 2006; Lejon et al., 2003). Trypanosomal genetics (including trypanosomal intrinsic characteristics), human immune polymorphisms and geographical locations among others have been shown to define the severity or mildness of HAT in different settings (Smith and Bailey, 1997; Truc et al., 1997; Jamonneau et al., 2000; MacLean et al., 2004; Courtin et al., 2008). As the parasite devices ways to evade the human immune system in the light of the current burden of HAT, this review highlights the challenges posed by human African trypanosomes to the host immune system while underscoring the need to maximize the use of currently available control measures to keep the incidence of the disease low while further research for the development of a suitable vaccine continues.

\section{The burden of HAT}

There are about 200 active foci of trypanosomal infection in 36 countries in subSaharan Africa with more than 60 million people at risk of infection (WHO, 2001). The location of endemic foci of HAT follows the patchy distribution of tsetse flies found in a belt that stretches south of the Sahara and north of the Kalahari Desert (Barrett et al., 2003). Twenty-four (24) of the 36 endemic countries for HAT are reported to transmit $T$. $b$. gambiense, while T. $b$. rhodesiense is transmitted in 13 countries. Recently published data showed that between 1997 and 2006, T. b. gambiense represent $97 \%$ of the total new cases while the remaining $3 \%$ was due to T. $b$. rhodesiense (Simarro et al., 2008).

HAT is caused by subspecies of the haemoflagellate protozoan parasite, Trypanosoma brucei (T. b. gambiense and T. $b$. rhodesiense) which are transmitted by tsetse flies belonging to the genus Glossina. Sleeping sickness has a devastating impact on human health and economic prosperity. Greater than 500,000 cases and 70,000 deaths per year occur as a result of the parasite (WHO, 2001). The case fatality rate in untreated patients is $100 \%$; and this combined with the focal nature of the disease, means that the disability adjusted life years (DALYS) averted per infection cured or prevented are very high (WHO, 2001).

The animal subspecies of Trypanosomes, T. b.brucei, T. congolense and T. vivax (which causes "nagana" in cattle) is a major hindrance to cattle production in SubSaharan Africa (Hill et al., 2005). Indeed, the major economic losses from the animal form of this disease are due to cattle death and morbidity, diagnosis and treatment costs, reduction in meat and milk production by infected animals and the reduction of livestock production areas (Swallow, 1998; Olufemi et al., 2007). This clearly translates to the nutritional deficiency and poverty of the inhabitants in HAT endemic areas. Thus both 
human and animal trypanosomiasis are culprits in the development of the African continent, and are seen as an important impediment in the establishment of a flourishing agriculture to provide the highly desired food security and to enhance sustainable growth and healthy populations (Simarro et al., 2008).

Currently, there is no vaccine to prevent HAT and the burden of the disease is complicated by huge spending on trypanocidal drugs and challenges in vector control. Consequently, trypanosomiasis has reportedly cost over \$4 billion United States Dollars each year in direct costs and production (Hannotte et al., 2003). The burden of HAT is still high in Angola, Democratic Republic of Congo, Uganda and Sudan; and this high burden is directly related to factors such as decreased surveillance and control activities, war, parasite, and Glossina resistance to drugs and insecticide, migrations of population, and host disease susceptibility (Courtin et al., 2008; Simarro et al., 2008). Indeed, there is a current risk of overlap of $\mathrm{Tbg}$ (from Sudan) and $\mathrm{Tbr}$ (from Uganda) and this may complicate the burden of disease in these areas (Picozzi et al., 2005).

The current tools (diagnosis and treatment) available for the control of HAT in Africa have two technical impediments: lack of sensitive diagnostic test and of new, cheaper, and safe drugs that are easy to administer, which consequently limits the full participation of the health system (Simarro et al., 2008).

\section{Development of trypanosomes and clinical advancement in man \\ HAT takes two forms ( $T b g$ and Tbr),} and this depends on the parasites involved. Tbg is found in West and Central Africa and causes chronic infection. In this case, an infected individual for several months or years do not present with major signs or symptoms. These symptoms which include: severe headache, continuous fever, sleep disturbances, alteration of mental state and neurological abnormalities, occur at the advanced stage, with the involvement of the Central Nervous System (WHO, 2001). Tbr, found in Eastern and Southern Africa, causes an acute infection. In this case, signs and symptoms (chancre, intermittent headache and fevers, pruritus, and adenopathies) are seen after a few weeks or months and this marks the first stage of the infection (Barret et al., 2003).

Generally, the infection begins with the bite of an infected tse-tse fly, and is initially localized in the chancre at the bite site before spreading to the blood and lymph to commence the first stage of the infection - the haemolymphatic stage (stage I)(Courtin et al., 2008). This is followed by the invasion of cerebrospinal fluid leading to the second stage - the meningoencephalic phase (stage II).

The resulting disease (HAT) therefore, is dependent on the host susceptibility status and the strain's virulence (MacLeans et al., 2007). The dichotomy that exists between $\mathrm{Tbg}$ and $T b r$, does not fully represent the disease process (Smith and Bailey, 1997; Truc et al., 1997; Jamonneau et al., 2000; MacLean et al., 2004; Courtin et al., 2008). Patients infected with $T b g$ could present with a spectrum of clinical manifestations from asymptomatic (Jamonneau et al., 2000) to acute infections similar to $\mathrm{Tbr}$ trypanosomiasis (Truc et al., 1997). On the other hand, cases of Tbr chronic infections (similar to Tbg) have also been reported (Smith and Bailey, 1997; MacLeans et al., 2004).

The different responses to the infection are determined by factors from host, the parasite and the environment, with the immune system and its genetic control being chief contributors (MacLeans et al., 2007). The identification of two trypanosomiasis foci with diametrically different disease profiles in Uganda and Malawi (both $\mathrm{Tbr}$ foci), that resulted in mild and severe forms of HAT made MacLeans et al. (2004) to conclude that host responses may result either from different virulence phenotypes of trypanosomes (northern and southern within the East and Southern African type) or from immune response polymorphisms in the different host populations.

\section{Human trypanosome genetics}

Morphologically, $\mathrm{Tbg}$ and $\mathrm{Tbr}$ are indistinguishable under the microscope. However, they are different genetically. While Tbg has a characteristic TgsGP gene, Tbr has the human serum-resistance associated gene (SRA) (Gibson et al., 2002; Picozzi et al., 2005) and this plays a functional role in 
permitting human infectivity (Vanhamme et al., 2003). While Tbg is somewhat stable, allelic polymorphisms in the SRA gene and its genomic environment occur in $\mathrm{Tbr}$ isolates from East and South African countries (Gibson et al., 2002; Gibson and Ferris, 2003; MacLean et al., 2004).

Two spatially distinct foci of HAT $(T b r)$ in the Tororo and Soroti area of Eastern Uganda have been described. $\mathrm{Tbr}$ in these two areas were shown to be genetically distinct using 6 microsatellite and 1 minisatelite polymorphic markers, which differentiated the disease progression and host immune response (MacLean et al., 2007). Interestingly, infections with the Tororo genotype showed an increased frequency of progression to severity of the meningoencephalitic stage and higher IFN- $\gamma$ concentration compared to those with the Soroti genotype. Earlier, two disease profiles of $\mathrm{Tbr}$ in a study in Uganda (acute profile and rapid progression to the late stage meningoencephalitic infection described) and Malawi (chronic profile with infection lasting for several months described) were associated with markedly different levels of tumor necrosis factor alpha (TNF- $\alpha)$ and transforming growth factor $\beta$ (TGF- $\beta$ ) in plasma (MacLean et al., 2004). In Uganda but not in Malawi, early stage (TNF- $\alpha$ ) was elevated, while in Malawi but not Uganda, early (TGF- $\beta$ ) was elevated. MacLean et al. (2004), therefore inferred that rapid disease progression in Uganda was associated with TNF- $\alpha$ mediated inflammatory pathology, whereas in the milder disease recorded in Malawi may be ameliorated by counterinflammatory cytokines. SRA gene and immune response polymorphisms in the various host populations were elucidated in this study.

The influence of human genetic polymorphisms, particularly those located in cytokine genes, in the control of Tbg infection in Côte D'Ivore (Ivory Coast) and Democratic Republic of Congo (DRC) was reported recently (Courtin et al., 2006, 2007, 2008). On the one hand, it was shown that significant association between polymorphisms located in the IL6 and ILIO genes decreased risk of developing HAT and, on the other hand, a significant association between polymorphisms located in ILI- $\alpha$ and $T N F \alpha$ genes increased risk of developing the disease (Courtin et al., 2006, 2007).

It has also been suggested that in a Congolese foci, pygmy population were less susceptible to the Tbg than the Bantou group (Vincendeau et al., 1999).

Response of $T$. brucei (rhodesian and gambian subspecies) to the human immune system

Antigenic Variation

African trypanosomes have co-evolved mechanisms to regulate every major aspect of host innate and acquired immunity in order to survive. Antigenic variation is central to the success of African trypanosomes and other eukaryotic, bacterial and viral pathogens (McCulloch, 2004). The surface coat of trypanosomes is made up of a monolayer of approximately $10^{7} \quad$ VSG homodimers (Borst,2002), tethered by GPI anchors to the extracellular surface of the trypanosome plasma membrane, forming a dense glycoprotein coat that protects the membrane of the parasite from the host environment (Cross, 1975; Cardoso de Almeida et al.,1983). Trypanosomes undergo antigenic variation by changing this surface coat when a new VSG gene, from a library of up to $10^{3}$ different surface antigen genes, is expressed effectively ensuring that the host immune response does not fully eliminate the organisms. This cyclical pattern of trypanosome outgrowth and variant specific elimination may continue throughout infection until the host succumbs (Cardoso de Almeida et al., 1983).

T. brucei grow in the bloodstream and tissue fluids of mammals, and are subjected to specific and non-specific immune attack (Cross, 1996; Donelson, 2003). To hide variant surface antigens from the immune response, trypanosomes shroud themselves in the variant surface glycoprotein (VSG) coat.

The molecular identity of these VSGs changes periodically, allowing a part of the parasite population to avoid antibodymediated killing throughout an infection. Stringent allelic exclusion, directed by an extranucleolar protein complex containing RNA polymerase I, ensures that only one VSG gene is transcribed at any given time from a chromosome telomere (Navarro and Gull, 2001). Thus, by switching VSG genes 
and expressing a new surface coat, trypanosomes evade B- and Th-cell immune responses directed against VSG epitopes displayed in the former surface coat. The end result is that new variant antigenic types (VATs) populate the vascular and extravascular tissues and perpetuates the infection, whereas trypanosomes expressing the former coat are destroyed. There is therefore a dynamic anti-VSG response by the $\mathrm{B}$ and $\mathrm{T}$ helper cells, which is directed against every new epitope formed by the parasite (Cardoso de Almeida et al., 1983).

The VSG coat acts as a macromolecular sieve, preventing the approach of complement components to the otherwise sensitive plasma membrane (Ferrante and Allison, 1983). There are several hundred genes encoding immunologically distinct VSG variants; switching the expression of these genes allows that parasite population to evade the specific immune response through antigenic variation (Vanhamme et al., 2001). Variant antigen type (VAT) switching involves the differential expression of VSG genes and occurs, at the population level within the host, at a rate of about $10^{-2}$ switches per cell per generation (Barry and McCulloch, 2001). Some workers have proposed that there is an order in the switching of VSG genes which is best described as semi-predicatable (Barry and McCulloch, 2001; Morrisona et al., 2005) and the type of locus occupied by a VSG gene can influence the timing of its activation (Pays, 2006; Taylor and Rudenko, 2006).

Dubois et al. (2005) observed during the transient period of switching VSGs ( 48h), when two surface coats are simultaneously displayed, trypanosomes fail to directly activate B cells until coat homogeneity is achieved. This gives the parasite a chance to survive for sometime before the development of immune response to the new surface coat.

\section{Cleaning of parasite's surface proteins}

The trypanosome is sheathed in a dense protective coat of VSG. The only gap in this armour is the flagellar pocket, a small invagination of the plasma membrane at the base of the flagellum where nutrients are taken up and molecules are secreted. Processes such as endocytosis, exocytosis and surface membrane turnover in general are highly polarised and restricted entirely to the flagellar pocket (Vaughan and Gull, 2003).

The flagellar pocket membrane accounts for about $1 \%$ of the surface area of the parasite (Wellcome Trust, 2005).The parasite also uses the flagellar pocket to take up and degrade antibodies. Within minutes of antibody binding, the parasite cleans its surface by transferring the bound antibody to the flagellar pocket where it is rapidly internalised and degraded. This provides the parasite a means of evading destruction by antibodies. If the parasite is prevented from grooming itself in this way, it will not survive (WellcomeTrust, 2005).

\section{Regulation of host immune system}

One way trypanosomes manipulate the host immune response during infection is by reducing the effectiveness of IFN- $\gamma$ activation of macrophages. Exposure of macrophages to soluble VSG (sVSG) prior to IFN- $\gamma$ treatment has been shown to result in marked reduction in cell activation events including inducible nitric oxide synthase (iNOS) gene transcription, nitric oxide (NO) release and IL-6 (Coller et al., 2003; Mansfield and Paulnock, 2005). Thus the timing of macrophage exposure to these 2 different activating agents, IFN- $\gamma$ and VSG, may determine whether macrophages are fully activated or down regulated.

Recently, it was demonstrated in animal models that in addition to antigenic variation, trypanosome infection also causes significant loss of various B cell populations and an extensive remodeling of the splenic micro-architecture (Radwanska et al., 2008). These changes were reported to (a) disable the host's capacity to mount a proective antiparasite antibody responses, (b) prevent the development of effective B-cell memory against encountered variant antigenic parasite types (VATs), and (c) abrogate vaccineinduced protective response to non-related pathogen such as Bordettela pertusis in a setting where commercially available human vaccine for diphtheria, tetanus, and $B$. pertusis (DTPa) was used in experimental mouse model (Radwanska et al., 2008).

\section{Host response to trypanosomal infection Non-immune response}

The infectivity of African trypanosome species in humans appears to be defined by 
their susceptibility to two lytic factors in normal human serum called trypanosome lytic factors 1 and 2 (TLF1 and TLF2). Trypanosome lytic factor 1 (TLF1) is a subclass of human high density lipoprotein (HDL) containing apolipoprotein A-I (apoAI), apolipoprotein L-I (apoL-I), and haptoglobin-related protein (Hpr) (Raper et al., 1999). TLF2 is a $1,000-\mathrm{kDa}$ protein complex containing mainly immunoglobulin M, apoA-I, and Hpr but less than 1\% detectable lipid (Raper et al., 1999). Trypanosoma brucei brucei, the causative agent of nagana in cattle and which can also infect a wide range of mammals, is unable to infect humans because it is susceptible to the innate cytotoxic activity of normal human serum (Hager et al., 1994; Vanhamme et al., 2003).

Different mechanisms of lysis of trypanosomes by TLF have been proposed by different workers. Oli et al. (2006) suggested that TLF kills $T$. $b$. brucei following receptor binding, endocytosis, and lysosomal localization. Thus the auto-digestion of the organism by the contents of the ruptured lysosomes leads to cell lysis. Molina-Portela et al. (2005) proposed that TLF1 acts by forming cation-selective pores in membranes leading to osmotically driven swelling and lysis of trypanosomes. The net influx of both $\mathrm{Na}^{+}$and $\mathrm{Cl}^{-}$create an osmotic imbalance that leads to passive water diffusion. This loss of osmoregulation results in cytoplasmic vacuolization, cell swelling and ultimately trypanosome lysis.

T. b. rhodesiense and T. b. gambiense, which are morphologically and physiologically indistinguishable from $T . b$. brucei, are resistant to TLF-mediated killing. Human infectivity by $T . \quad b$. rhodesiense correlates with the expression of a serum resistance-associated (SRA) gene which encodes the SRA protein that is able to resist TLF killing (Raper et al., 2001; Oli et al., 2006). Vanhamme et al. (2003) proposed that apoL-I is the trypanosome lytic factor of normal human serum, and that SRA protein confers resistance to lysis by interaction with apoL-I in the lysosome. The mechanism underlying virulence in human African trypanosomiasis are still poorly understood, however, MacLean et al. (2004) reported the presence of SRA gene in all 231 patients with sleeping sickness outbreak from $T$. rhodesiense in East Uganda and TgsGP gene in $T$. b. gambiense confirming that the SRA gene is specific for Tbr. The difference observed in host responses in this study (MacLean et al., 2004) and several other studies may be due to different virulence phenotypes or could be as a result of polymorphism in the different host population (Mansfield and Paulnock, 2005; Courtin et al., 2008).

\section{Innate immune response}

Macrophages comprise the backbone of the host innate immune response, a key element in defense against parasitic infection. Macrophages are present in all tissues and possess the ability to recognize and eliminate many microbes. Pathogen recognition is mediated by the binding of specific microbial molecules to membrane pattern recognition receptors, such as the toll-like receptors (TLRs) on macrophages and dendritic cells, resulting in cellular activation (Mansfield and Paulnock, 2005). Receptor-mediated activetion of macrophages represents one of the first events in the innate immune response to many microbial infections, leading to the production of pro-inflammatory cytokines that initiate an inflammatory response and affect the downstream development of activated T cells. In addition to their role as antigen presenting cells (APCs), which allows the adaptive immune system to become responsive to the invading pathogen, macrophages produce products that have both autocrine and paracrine effects that serve to amplify the innate and adaptive immune responses (Mansfield and Paulnock, 2005).

Macrophages can also be stimulated to release reactive oxygen and nitrogen species, which have been demonstrated to have direct pathogen cytostatic and cytotoxic effects (Kaushik et al., 1999; Gobert et al., 2000). Thus, a coordinated macrophage response is essential in the initiation and maintenance of a protective host response during trypanosome infection.

Both soluble VSG (sVSG) and membrane form VSG (mfVSG) have similar macrophage activating capabilities in terms of inducing TNF- $\alpha$, IL- 6 and NO production, but there may be subtle differences in the comparative ability of mfVSG to more effectively induce IL-1 and IL-2 (Schofield 
and Tachado, 1996; Magez et al., 1998). Early during infection, a strong and effective host immune response is mounted against the parasite that includes B cell and Th1 cell stimulation by VSG determinants in addition to macrophage activation, resulting in destruction of trypanosomes expressing the target VSG in murine African trypanosomiasis (Hudson et al., 1976; Grosskinsky et al., 1983).

Pathogen-recognition receptors such as TLR9 on macrophages and other cells recognize foreign DNA containing cytosinephosphate-guanine $(\mathrm{CpG})$ sub-sequences and generate strong $\mathrm{NF \kappa B-dependent} \mathrm{signaling}$ responses that result in production of cytokines. Trypanosomes contain $\mathrm{CpG}$ motifs in their nuclear (but not kinetoplast) DNA (Mansfield and Paulnock, 2005). Early cytotoxic effects mediated by sVSG-activated macrophages result in the release of trypanosome DNA and subsequent B- and Th1 cell-mediated immune responses potentiate such release and subsequent amplification of cell activation. Highly purified trypanosome DNA have been shown to stimulate macrophages to produce IL-1 $\beta$, TNF- $\alpha$, IL-6, GM-CSF, prostaglandin $\mathrm{E}_{2}$, NO and IL-12 (Mansfield and Paulnock, 2005). Interestingly, HAT infection with T.b. gambiense cross microvascular endothelial cells (BMECs), a human blood brain barrier (BBB) model system at a much greater rate efficiency than do T.brucei because T.b.gambiense display high levels of cathepsin L-like cysteine proteases. Nikolskaia et al. (2006) reported that using cysteine protease inhibitors K11777 and CA074, evidence showed that cathepsin L-like brucipain is probably the cysteine responsible for blood brain barrier (BBB). This in their opinion plays an important role in transendothelial migration of the human brain. Recent animal model studies have also demonstrated that IFN- $\gamma$ plays a critical role in this process, by modulating endothelial basement membrane laminin expression and lymphocyte transmigration (Masocha et al., 2004; MacLean et al., 2007).

Acquired immune response

Cytokines produced by activated Th cells, primarily IFN- $\gamma$, provide additional activation signals for macrophages, unleashing effector functions that can destroy a wide range of intra- and extracellular microorganisms. Thus, macrophages play a dual role as the initiators of acquired responses and as a major effector component of cell-mediated immunity (Mansfield and Paulnock, 2005).

The host immune responses to African trypanosomes that are triggered by infection include both $\mathrm{B}$ and $\mathrm{T}$ lymphocyte responses to variant surface glycoprotein (VSG) molecules that comprise the surface coat structure. These acquired immune responses impact on parasite survival in the vascular and extravascular tissue compartments of the infected host; furthermore, the magnitude and quality of VSG-specific B and Th cell responses largely are shaped by interactions of trypanosomes and their molecules with cells of the innate immune system (Mansfield and Paulnock, 2005).

Trypanosomes activate both T-helper (Th) cell-independent and Th-cell-dependent B-cell responses to the VSG molecule, with the result that antibodies to exposed VSG epitopes of the surface coat efficiently clear the organisms from the blood. Coincidentally, Th lymphocytes are activated by exposure to VSG peptide-MHC II complexes on antigenpresenting cells (macrophages and dendritic cells) to produce a highly polarized type 1 cytokine response. This response includes the release of IFN- $\gamma$, which activates tissue macrophages to produce a number of trypanocidal factors such as reactive nitrogen intermediates (RNI), reactive oxygen intermediates (ROI), TNF- $\alpha$ and perhaps other molecules that are capable of destroying trypanosomes in the extravascular tissues. (Mansfield and Paulnock, 2005)

Maximum host resistance to trypanosomes requires both the B cellmediated antibody response and the Th1 cell responses leading to the production of IFN- $\gamma$. IFN- $\gamma$ induce macrophage trypanolytic and trypanostatic activities (Daulouede et al., 1994; Hertz et al., 1998). High levels of both tumor necrosis alpha (TNF- $\alpha$ ) and interleukin 10 (IL-10) have been associated with trypanosomal infection (MacLean et al., 2001; Lejon et al., 2003).

Increased intrathecal cytokine levels and blood-cerebrospinal fluid (CSF) barrier function were studied among $T b r$-infected patients in south-east Uganda (MacLean et al., 
2006). In this study, the CSF concentration of the cellular immune activation marker neopterin (a general marker for activation of the cellular immune system which is released by activated macrophages and other cells of the monocyte lineage including astrocytes [Fuchs et al., 1988]) and cytokines IL-6 and IL-10 were increased over control and post treatment levels in all patients, with maximal levels observed in late stage (meningoencephalitic) individuals. Furthermore, the CSF/serum concentration quotients indicated that IL-10 and neopterin were derived from CNS synthesis in at least $25 \%$ of the 91 patients while blood-CSF barrier dysfunction occurred in $64 \%$ of late stage patients but not in early stage patients (MacLean et al., 2006). The authors however noted that while the high level of neopterin observed in the late-stage patient CSF is indicative of widespread cellular activation, the finding of IL- 6 and IL-10 suggested that counter-inflammatory cellular responses may be important in the regulation of neuropathogenesis in late stage HAT.

Some studies have demonstrated that trypanosome-infected mice with a resistant genetic background, but lacking the IFN- $\gamma$ gene, were as susceptible as infected scid mice, despite the fact that they made VSGspecific Abs that controlled parasitemia in the blood. These data reveal that IFN- $\gamma$ is a crucial element of the host response to these parasites (Hertz et al., 1998). It is well established that the IFN- $\gamma$-mediated macrophage activation include the enhancement of MHC class II expression leading to enhanced Th cell response and the stimulation of microbicidal factors such as nitric oxide (NO). These two functional activities also appear to play key roles in the host response to trypanosome infection (Hertz and Mansfield, 1999; MacLean et al., 2001). However, during infection with African trypanosomes, parasite-derived molecules also capable of modulating macrophage activation are released. Among these are the glycosylinositolphosphate soluble VSG (GIPsVSG) molecules, which are cleaved from the trypanosome membrane by the action of glycosylphosphatidylinositol-phospholipase C (GPI-PLC) (Coller et al., 2003).

Coller et al. (2003) also showed that during early trypanosome infection (days 1-
15), a robust IFN- $\gamma$ response is made, correlating with the rise and fall of the first wave of parasitaemia and coincide with a moderate release of GIP-sVSG. In contrast, during late stage infection (days 35-55), IFN$\gamma$ production was no longer detectable in mice exhibiting sustained high levels of parasitaemia and abundant levels of GIPsVSG are present.

Clinical evidence of the involvement of IFN- $\gamma$ in the initiation and severity of meningoencephalitic sleeping sickness was described by MacLean et al., (2007), in Tbr infection in Uganda. Increased frequency of plasma IFN- $\gamma$ concentration correlated with the progression of rapid and acute sleeping sickness among a particular tribe in Uganda. Generalized up-regulation of plasma IL1 $\beta$, TGF- $\beta$, and IL-10 in all patients for both Tbg and $\operatorname{Tbr}$ (MacLean et al., 2007).

\section{The way forward}

The way forward for HAT is to eliminate it. This has already been proposed in the 1997 World Health Assembly elimination resolution (WHO, 2001). This resolution led to the scaling up of field activities with subsequent reduction in new cases of HAT and the participation of both public and private contributors (Simarro et al., 2008). A Pan African Tsetse and Trypanosomiasis Eradication Campaign (PATTEC) is already on in some African countries with encouraging results. Nevertheless, there is need to integrate control measures with other disease control activities for cost-effectiveness and sustainability; institution of new approaches to surveillance and control; development of new diagnostic tools (including tools for staging the disease); development of new drugs and vaccines; and the development of effective vector control strategy. The review by Simarro and colleagues has addressed these points and in addition, continuous research in HAT endemic countries was proposed recently (Simarro et al., 2008).

\section{Conclusion}

Human infection by T.b. gambiense and T.b. rhodesiense is made possible by their expression of serum resistance associated protein which nullifies the lytic action the normal human serum has on the cattle 
pathogen, $T . b$. brucei. In order to successfully establish an infection, the trypanosomes evade complete destruction by the host immune system primarily by switching the variant surface glycoprotein on their surface membrane. Trypanosomes expressing mosaic mfVSG escape immune recognition, hence giving the parasite opportunity to survive.

Trypanosome-derived mfVSG, sVSG and $\mathrm{CpG}$ DNA all regulate macrophage activation and the production of cytokines like TNF- $\alpha$, IL-6 and IL-12 as well as other factors such as ROI and RNI which can destroy the parasites. Macrophages play a central role in the host immune response to the presence of trypanosomes in the vascular and extravascular systems, activating both the innate and acquired immune response. Trypanosomes activate both Th-dependent and Th-independent B-cell responses to the VSG molecules which effectively clear the parasites expressing those VSGs. VSGs are anchored on parasite membrane by GPI molecules. The development of specific inhibitors of GPI biosynthetic enzymes could be used as therapeutic agents of trypanosomiasis. It is worthy to note that adequate knowledge of the host genetic studies on trypanotolerance in mice, cattle and human should be of interest to researchers. Generally, trypanosome strains, trypanosomal genetic polymorphisms, human immune response polymorphisms, the environment, among other factors are important elements that describe the severity and mildness of HAT. Understanding these relationships between the parasite and host genetic make-up may likely contribute new insights into how the parasites can be controlled and provide useful information on the pathophysiology of the parasite, which could help in discovering new therapeutic agents or in trypanosome vaccine development in order to reduce new cases in endemic foci. Finally, early and prompt diagnosis and treatment, and the institution of vector control measures will be helpful in reducing the incidence of HAT.

\section{REFERENCES}

Barrett MP, Burchmore RJ, Stich A, Lazzari JO, Frasch AC, Cazzulo JJ, Krishna S. 2003. The trypanosomiasis. Lancet, 362: 1469-1480.
Barry JD, McCulloch R. 2001. Antigenic variation in trypanosomes: enhanced phenotypic variation in a eukaryotic parasite. Adv. Parasitol., 49: 1-70.

Borst P. 2002. Antigenic variation and allelic exclusion. Cell, 109: 5-8.

Cardoso de Almeida ML, Turner MJ. 1983. The membrane form of variant surface glycoproteins of Trypanosoma brucei. Nature, 302: 349-352.

Coller SP, Mansfield JM, Paulnock, DM. 2003. Glycosylinositolphosphate soluble variant surface glycoprotein inhibits IFN$\gamma$-Induced nitric oxide production via reduction in STAT1 phosphorylation in african trypanosomiasis. J. Immunol., 171: 1466-1472.

Courtin D, Argiro L, Jamonneau V, N'dri L, N'guessan P, Abel L, Dessein A, Cot M, Laveissiere C, Garcia A. 2006. Interest of tumor necrosis factor- alpha 308G/A and interleukin-10-592CA polymorphisms in human african trypanosomiasis. Infect. Genet. Evol., 6: 123 - 129.

Courtin D, Miller J, Jamonneau V, Yeminanga CS, Kumeso, VK, Bilengue CM, Betard C, Garcia A. 2007. Association between human african trypanosomiasis and the IL-6 gene in a congolese population. Infect. Genet. Evol., 7: 60 - 68.

Courtin D, Berthier D, Thevenon S, Dayo GK, Garcia A, Bucheton B. 2008. Host genetics in african trypanosomiasis. Infect. Genet. Evol., 8: 229-238.

Cross GA. 1975. Identification, purification and properties of clone-specific glycoprotein antigens constituting the surface coat of Trypanosoma brucei. Parasitol., 71(3): 393-417.

Cross GA. 1996. Antigenic variation in trypanosomes: secrets surface slowly. Bioessays, 18: 283-291.

Daulouede PS, Okomo-Assoumou, MC, Labassa M, Fouquet C, Vincendeau P. 1994. Defense mechanisms in trypanosomiasis. Bull. Soc. Pathol. Exot., 87: $330-332$.

Donelson JE. 2003. Antigenic variation and the african trypanosome genome. Acta Trop., 85: 391-404.

Dubois ME, Demick KP, Mansfield JM. 2005. Trypanosomes expressing a mosaic variant surface glycoprotein coat escape 
early detection by the immune system. Infect. Immun., 73: 2690-2697.

Ferrante A, Allison AC. 1983. Alternative pathway activation of complement by African trypanosomes lacking a glycolprotein coat. Parasite Immunol., 5: 491498.

Fuchs D, Hausen A, Reilbnegger G, Werner ER, Dierich MP, Watcher H. 1988. Neopterin as a marker of a activated cellmediated immunity: Applications on HIV infection. Immunol. Today, 9: 150 - 155.

Gibson W, Backhouse T, Griffiths A. 2002. The human serum associated gene is ubiquitous and conserved in Trypanosoma brucei brucei throughout East Africa. Infect. Genet. Evol., 1: 207214.

Gibson, W, Ferris V. 2003. Conservation of the genomic location of the human serum resistance associated gene in Trypanosome brucei rhodisiense. Mol. Biochem. Parasitol., 130: 159-162.

Gobert AP, Daulouede S, Lepoivre M, Boucher JL, Bouteille B, Buguet A, Cespuglio R, Veyret B, Vincendeau P. 2000. L-Arginine availability modulates local nitric oxide production and parasite killing in experimental trypanosomiasis. Infect. Immun., 68: 4653.

Grosskinsky CM, Ezekowitz RA, Berton G, Gordon S, Askonas BA. 1983. Macrophage activation in murine african trypanosomiasis. Infect. Immun., 39: 1080-1086.

Hager M, Pierce MA, Moore DR, Tytler EM, Esko JD, Hajduk SL. 1994. Endocytosis of a cytotoxic human high density lipoprotein results in disruption of acidic intracellular vesicles and subsequent killing of African trypanosomes. J. Cell Biol., 126: 155-67.

Hannotte O, Ronin Y, Agaba M, Nilsson P, Gelhaus A, Horstmann R, Sugimoto Y, Kemp S, Gibson J, Korol A, Soller M, Teale A. 2003. Mapping of quantitative trait loci controlling trypanotolerance in a cross of tolerant West African N'Dama and susceptible East African Boran cattle. Proc. Natl. Acad. Sci. USA, 100: 74437448.

Hertz CJ, Filutowicz H, Mansfield JM. 1998. Resistance to the African trypanosomes is
IFN- $\gamma$ dependent. J. Immunol., 161: 67756783.

Hertz CJ, Mansfield JM. 1999. IFN- $\gamma$ dependent nitric oxide production is not linked to resistance in experimental African trypanosomiasis. Cell. Immunol., 192: 24-32.

Hill EW, O'gorman GM, Agaba M, Gibson JP, Hanotte O, Kemp SJ, Naessens J, Coussens PM, Machugh DE. 2005. Understanding bovine trypanosomiasis and trypanotolerance: the promise of functional genomics. Vet. Immunol. Immunopathol., 105: 247 - 258.

Hudson KM, Byner C, Freeman J, Terry RJ. 1976. Immunodepression, high IgM levels and evasion of the immune response in murine trypanosomiasis. Nature, 264: 256-258.

Jamonneau V, Garcia A, Frezal JL, N'guessan P, N'dri L, Sanon R, Laveissiere C, Truc P. 2000. Clinical and biological evolution of human trypanosomiasis in Côte d'Ivore. Ann. Trop. Med. Parasitol., 94: $831-835$.

Kaushik RS, Uzonna JE, Gordon JR, Tabel H. 1999. Innate resistance to Trypanosoma congolense infections: differential production of nitric oxide by macrophages from susceptible BALB/c and resistant C57BL/6 mice. Exp. Parasitol., 92: 131-143.

Lejon V, Lardon J, Kenis G, Pinoges L, Legros D, Bisser S, N'Siesi X, Bosmans E, Buscher P. 2003. Interleukin (IL)-6, IL-8 and IL-10 in serum and CSF of Trypanosoma brucei gambiense sleeping sickness patients before and after treatment. Trans. R. Soc. Trop. Med. Hyg., 96: 329 - 333.

MacLean L, Odiit M, Sternberg JM. 2001. Nitric oxide and cytokine synthesis in human African trypanosomiasis. J. Infect. Dis., 184: 1086-1090.

MacLean L, Chisi JE, Odiit M, Gibson WC, Ferris V, Picozzi K, Sternberg JM. 2004. Severity of human african trypanosomiasis in East Africa is associated with geographical location, parasite genotype and host inflammatory cytokine response profile. Infection Immunity, 72: 70407044.

MacLean L, Odiit M, Sternberg JM. 2006. Intrathecal cytokine response in human 
African trypanosomiasis. Trans. R. Soc. Trop. Med. Hyg., 100(3): 270 -275.

MacLean L, Odiit M, MacLeod A, Morrison L, Sweeney L, Cooper A, Kennedy PGE, Sternberg JM. 2007. Spatially and genetically distinct African trypanosome virulence variants defined by hostinterferon-gamma response. J. Infect. Dis., 196: 1620-1628.

Magez S, Stijlemans B, Radwanska M. 1998. The glycosylinositol-phosphate and dimyristoylglycerol moieties of the glycosylphosphatidylinositol anchor of the trypanosome variant specific surface glycoprotein are distinct macrophageactivating factors. J. Immunol., 160: 1949-1956.

Mansfield JM, Paulnock DM. 2005. Regulation of innate and acquired immunity in African trypanosomiasis. Parasite Immunol., 27: 361-371.

Masocha W, Robertson B, RottenbergME, Mhlanga J, Sorokin L, Kristesson K. 2004. Cerebral vessel laminins and IFNgamma define Trypanosoma brucei penetration of the blood-brain barrier. $J$. Clin. Invest., 114: 689-694.

McCulloch R. 2004. Antigenic variation in african trypanosomes: monitoring progress. Trends Parasitol., 20: 117-121.

Molina-Portela MP, Lugli EB, Recio-Pinto E, Raper J. 2005. Trypanosome lytic factor, a subclass of high-density lipoprotein, forms cation-selective pores in membranes. Mol Biochem Parasitol., 144: 218-26.

Morrisona LJ, Majiwab P, Readc AF, Barry JD. 2005. Probabilistic order in antigenic variation of Trypanosoma brucei. Int. J. Parasitol., 35: 961-972.

Navarro M, Gull K. 2001. A pol I transcriptional body associated with VSG mono-allelic expression in Trypanosoma brucei. Nature, 414: 759-763.

Nikolskaia OA, de A Lima APC, Kim YV, Lonsdale-Eccies JD, Fukuma T, Scharfstein J, Grab DJ. 2006. Blood-brain barrier traversal by African trypanosomes requires calcium signaling induced by parasites cysteine protease. J. Clin. Invetig., 116(10): 2739-2747.

Oli MW, Cotlin LF, Shiflett AM, Hajduk SL. 2006. Serum resistance-associated protein blocks lysosomal targeting of trypanosome lytic factor in Trypanosoma brucei. Eukaryot Cell, 5: 132-139.

Olufemi RA, Illemobade AA, Laeinde EAO.2007. The impact of African animal trypanosomiasis and tsetse on the livelihood and well-being of cattle and their owners in the BICOT study area of Nigeria. Sci. Res. Essay, 2: 380-383.

Pays E. 2006. The variant surface glycolprotein as a tool of adaptation in African trypanosomes. Microbes Infect., 8: 930937.

Picozzi K, Fevre EM, Odiit M, Carrington M, Eisler MC, Maudlin I, Welburn SC. 2005. Sleeping sickness in Uganda: a thin line between two fatal diseases: $B M J, 311$ : $1238-1241$.

Radwanska M, Guirnalda P, Trez C, Ryffel B, Black S, Magez, S. 2008. Trypanosomiasis-induced B cell apoptosis results in loss of protective antiparasite antibody responses and abolishment of vaccine-induced memory responses. PLos Pathogens, 4(5): e1000078.

Raper J, Fung R, Ghiso J, Nussenzweig V, Tomlinson S. 1999. Characterization of a novel trypanosome lytic factor from human serum. Infect. Immun., 67: 19101916.

Raper J, Portela MP, Lugli E, Frevert U, Tomlinson S. 2001. Trypanosome lytic factors: novel mediators of human innate immunity. Curr Opin Microbiol., 4: 402408.

Schofield L, Tachado SD. 1996. Regulation of host cell function by glycosylphosphatidyl-inositols of the parasitic protozoa. Immunol. Cell Biol., 74: 555563.

Simarro PP, Jannin J, Cattand P. 2008. Eliminating Human African Trypanosomiasis: Where do we stand and what comes next? PLos Medicne, 5(2): 0174-0180.ican

Smith DH, Bailey JW. 1997. Human African trypanosomiasis in South-eastern Uganda: Clinical diversity and isoenzyme profiles. Ann. Trop. Med. Parasitol., 91: 851-856.

Swallow B, 1998. Impact of trypanosomiasis on African agriculture. PAAT position. paper. FAO-OMS-IAEA-OAU/IBAR; 47 
Taylor JE, Rudenko, G. 2006. Switching trypanosome coats: what's in the wardrobe ? Trends Genet., 22: $614-620$.

Truc P, Formentry P, Diallo PB, Komoin-oka C, Lauginie F.1997. Confirmation of two distinct classes of zymodemes of Trypanosoma brucei infecting man and wild mammals in Côte d'Ivore: suspected difference in pathogenicity. Ann. Trop. Med. Parasitol. 91: 951-956.

Vanhamme L, Paturiaux-Hanocq F, Poelvoorde P, Nolan D, Lins L, Van den Abbeele J, Pays A, Tebabi P, Xong H, Jacquet A, Moguilevsky N, Dieu M, Kane JP, De Baetselier P, Brasseur R, Pays E. 2003. Apolipoprotein L-I is the trypanosome lytic factor of human serum. Nature, 422: 83-87.

Vanhamme L, Pays E, McCulloch R, Barry JD. 2001. An update on antigenic variation in african trypanosomes. Trends Parasitol., 17: 338-343.

Vaughan S, Gull K, 2003. The trypanosome flagellum. J. Cell Sci., 116: 757-759.

Vincendeau P, Jauberteau-Marchan MO, Dauloudede S, Ayed Z. 1999. Immunology of African trypanosomiasis. In Progress in Human African Trypanosomiasis, Sleeping Sickness, Dumas M, Bouteille B, Buguet A (eds). Springer Verlag: Paris; $137-156$.

Wellcome T. 2005. Moving forward: The trypanosome flagellum. Http://www. wellcome.ac.uk/doc_wtx026059.html. [Accessed August 8, 2008].

WHO 2001. Report on African trypanosomiasis (sleeping sickness). Report of a Scientific Working Group, 4 - 8 June 2001. TDR/SWG/01: Geneva, Switzerland. 